\title{
Surface Defect Detection in a Tile using Digital Image Processing: Analysis and Evaluation
}

\author{
Foram Sanghadiya \\ M.Tech. in Computer Science \& Engineering \\ Institute of Technology, Nirma University \\ Ahmedabad - 382481
}

\author{
Darshana Mistry \\ Technical Associate, Software training \\ elnfochips Training \& Research Academy \\ Ahmedabad - 380060
}

\begin{abstract}
Quality control is imperative in any industry. Inspection of ceramic tile is done in conditions like noise, extreme temperature, and humidity. There are several stages through which we can maintain quality of a tile. These stages include inspection of color variation in a tile, chip offs in a tile, surface defects in a tile. This paper provides analysis of techniques which are useful to find surface defects like crack, blob, hole, variation in color, defect in corners, and pattern mismatch in tile which has pattern on it.
\end{abstract}

\section{Keywords}

Canny edge detection, image processing, thresholding, contrast stretching.

\section{INTRODUCTION}

In tile industry, production of tiles is in bulk. If inspection of a tile is done manually, it will take a lot of time. Also the making of tiles is actually done in automatic plant except quality check. So automation in inspection is one of the ways to reduce the time for inspection in tile industry. Inspection can be done in printing stage or later stage. While inspecting a tile, we may see certain defects in the surface of a tile like blob, pinhole, variation in color of a tile, crack, and chip offs in a tile, pattern mismatch, scratches etc. So after detecting the tile, we can apply some image processing steps to find these defects.

The fundamental stages comprising a Computer Vision system for digital image processing are: (a) Image acquisition, (b) Preprocessing, (c) Segmentation, (d) Feature extraction (representation and description), (e) Recognition interpretation and classification), and (f) Knowledge base [1]. From above, some of the stages can be removed according the real time digital image processing application.

\section{DEFECTS}

\subsection{Blob}

This type of defect can be found in tile due to existence of water in tile or proper humidity is not there.

\subsection{Pinhole}

This type of defect is very small. There are small holes in the surface of a tile, which are often undetectable by normal eyesight.

\subsection{Cracks}

This type of defect can be occurred during transmission of tiles or if high pressure is applied on a tile while manufacturing.

\subsection{Variation in Color}

If one part of the tile surface is colored with less amount of color than other, there is a possibility that this type of defect can be occurred.

\subsection{Chip offs}

This type of defect can be occurred due to collision with other object or while transmitting the tiles from one place to another place.

\subsection{Mismatch of pattern}

In printing phase, different pattern (other than mentioned pattern) was printed on a tile or placement of a tile is not correct then this type of defect can be found.

\section{RELATED STUDY}

For finding defects, various techniques can be applied on an image of a tile. Like thresholding, segmentation, subtraction of two images, clustering etc. In automatic inspection of apple quality, an image of a good apple is considered as a reference image, characteristic of the apple is obtained in terms of rules and image processing criteria by human expert. Then according to these evidences, other apples are examined and apple is classified in one of the predefined three categories [1]. Probabilistic neural network (PNN) can be applied. Different weights are given to the edges of this network and network is divided into four stages. Training set is given to the input stage and this set is passing through the pattern and summation layer followed by output layer. Set of tile images is taken as training set. This set is trained by passing through various stages like applying mean filter, resize the image, make histogram of R,G,B value, calculation of average contrast, detection of edges, form feature vectors, make feature classes based on feature vector class and train the classifier for detecting various defects on surface. Apply these feature vectors to PNN and take output as a defect [2].

Cracks, Spot, bumps, holes can be identified using various techniques. For that noise removal followed by binarization of image is done. Cracks can be identified based on figure aspect ratio and center of gravity equation. Spots can be identified using standard deviation using center of gravity, bumps and holes can be identified using edge detection techniques like Sobel, Prewitt, and Canny. Histogram equalization is used for setting contrast of the image [3]. Histogram equalization is very useful whenever in image; both background and foreground have bright colors or dark colors. Probability density function is used in histogram equalization [4].

Inspection of a tile can be done before kiln phase. Purpose of doing inspection before kiln phase is to reduce the number of defective tiles. Segmentation technique is used for finding corner defect. The purpose of segmentation is to divide an image into two regions: one contains background and corner and other contains tile part only. The challenging task in this 
algorithm is how to find two regions. For that different techniques are mentioned. Histogram subtraction is used for finding defects in corners of a tile. For that histograms of above two regions are taken. By subtracting one histogram from another, new histogram can be found. For deciding those two regions, mean standard deviation can be taken as threshold. Decision can be taken using following formula:

\section{If pixel $>T$ pixel $\epsilon$ Background plus Defects.}

\section{Else pixel $€$ Tile.}

After by using tile boundary points, unitary contour is found and using Hough transform straight lines for tiles are found. By comparing both of the contours tiles are classified into several classes. For classification some methods are compared. 1-NN Multiedit and condensing will give more accurate result than all other methods [5]. For contrast enhancement, adaptive histogram technique is used. Adaptive histogram technique will give better result than histogram equalization. Median filter and wiener filter can be used for noise removal. After detecting object using Sobel operator and region of interest using segmentation, by defining different threshold values cracks and blob type defects can be found. For removing unwanted lines, erosion and erode like morphological operations can be applied [6].

Crack in tile can be detect using local maxima, spot in tile can be found by modifying conventional linear filter, wigner filter can be used for cracks in textured tile. Using K-mean clustering, image is divided into chromatic category then if pixel is not classified in any category then we can conclude that tile has blob defect. To find pattern mismatch, present image of tile can be subtracted from reference image. If any difference can be found then we can say that tile has different pattern which is not acceptable [7].Canny gives efficient result than the sobel operator for detecting objects [8] [9] [10]. To detect blob, image can be complemented using inverse function followed by morphology operation, noise reduction. To detect spot, edge detection technique, morphology operation, noise reduction and smoothing can be applied sequentially. For detecting cracks same steps as detecting spot but in beginning image has to be converted in black and white [11].

\section{OBSERVATIONS}

From above analysis, we can say that for each defect, different technique is applicable to gain efficient result. So from below table, we can say which technique is more suitable for respective defect.

Table 1. Analysis of techniques for finding various defects

\begin{tabular}{|c|l|l|}
\hline Defect & \multicolumn{1}{|c|}{ Technique(s) } & \multicolumn{1}{c|}{ Remarks } \\
\hline Blob & $\begin{array}{l}\text { Segmentation using } \\
\text { particular threshold } \\
\text { value [6]. }\end{array}$ & $\begin{array}{l}\text { Difficult to find particular } \\
\text { threshold value. }\end{array}$ \\
\cline { 2 - 3 } & $\begin{array}{l}\text { Subtraction of } \\
\text { holograms of reference } \\
\text { tile image and current }\end{array}$ & $\begin{array}{l}\text { More resources are } \\
\text { required for hologram, } \\
\text { more time consuming. }\end{array}$ \\
\hline
\end{tabular}

\begin{tabular}{|c|c|c|}
\hline \multirow[t]{3}{*}{ Defect } & Technique(s) & Remarks \\
\hline & tile image [12]. & \\
\hline & $\begin{array}{l}\text { Using K-means } \\
\text { clustering } \\
\text { technique,chromatic } \\
\text { categories are } \\
\text { classified.If pixel is } \\
\text { not classified then } \\
\text { report an error. }\end{array}$ & $\begin{array}{l}\text { Machine learning } \\
\text { approach has to be } \\
\text { applied }\end{array}$ \\
\hline \multirow[t]{2}{*}{ Pinhole } & Thresholding & $\begin{array}{l}\text { Difficulty in finding } \\
\text { threshold value. }\end{array}$ \\
\hline & Line filter & $\begin{array}{l}\text { Modification in line filter } \\
\text { will give efficient result. }\end{array}$ \\
\hline \multirow[t]{4}{*}{ Cracks } & Local maxima & \multirow[t]{2}{*}{ More computation Time } \\
\hline & Wigner distribution & \\
\hline & Using classifier & Training set is required. \\
\hline & $\begin{array}{l}\text { Canny edge detector } \\
\text { with two threshold } \\
\text { values which can be } \\
\text { obtain from histogram } \\
\text { subtraction }\end{array}$ & $\begin{array}{l}\text { Good algorithm to } \\
\text { implement }\end{array}$ \\
\hline $\begin{array}{l}\text { Variation in } \\
\text { color }\end{array}$ & $\begin{array}{l}\text { Image subtraction then } \\
\text { edge detection } \\
\text { technique }\end{array}$ & Less accurate technique \\
\hline \multirow{3}{*}{$\begin{array}{l}\text { Chip offs } \\
\text { (Corner } \\
\text { defect) }\end{array}$} & $\begin{array}{l}\text { Region based } \\
\text { segmentation }\end{array}$ & Difficult to find regions \\
\hline & $\begin{array}{l}\text { Decision based on } \\
\text { angle of corner }\end{array}$ & \\
\hline & $\begin{array}{l}\text { Take image as a } \\
\text { matrix, some } \\
\text { preprocessing steps are } \\
\text { applied on matrix then } \\
\text { application of formula }\end{array}$ & $\begin{array}{l}\text { More time consuming } \\
\text { process }\end{array}$ \\
\hline
\end{tabular}

\section{RESULTS}

Results of some techniques are shown below. For Blob defect and variation in color, Canny edge detection technique is used. For Pinhole defect, first median filter is applied then threshold of 140 is taken for thresholding an image. For Crack defect, subtraction of reference image and current image followed by Canny edge detection is applied. In TABLE II, experimental results of different types of defects are shown. For experimental results, Visual Studio 2010 configured with openCV 2.4.8 is used. 
Table 2. Experimental results of detection of various types of defects

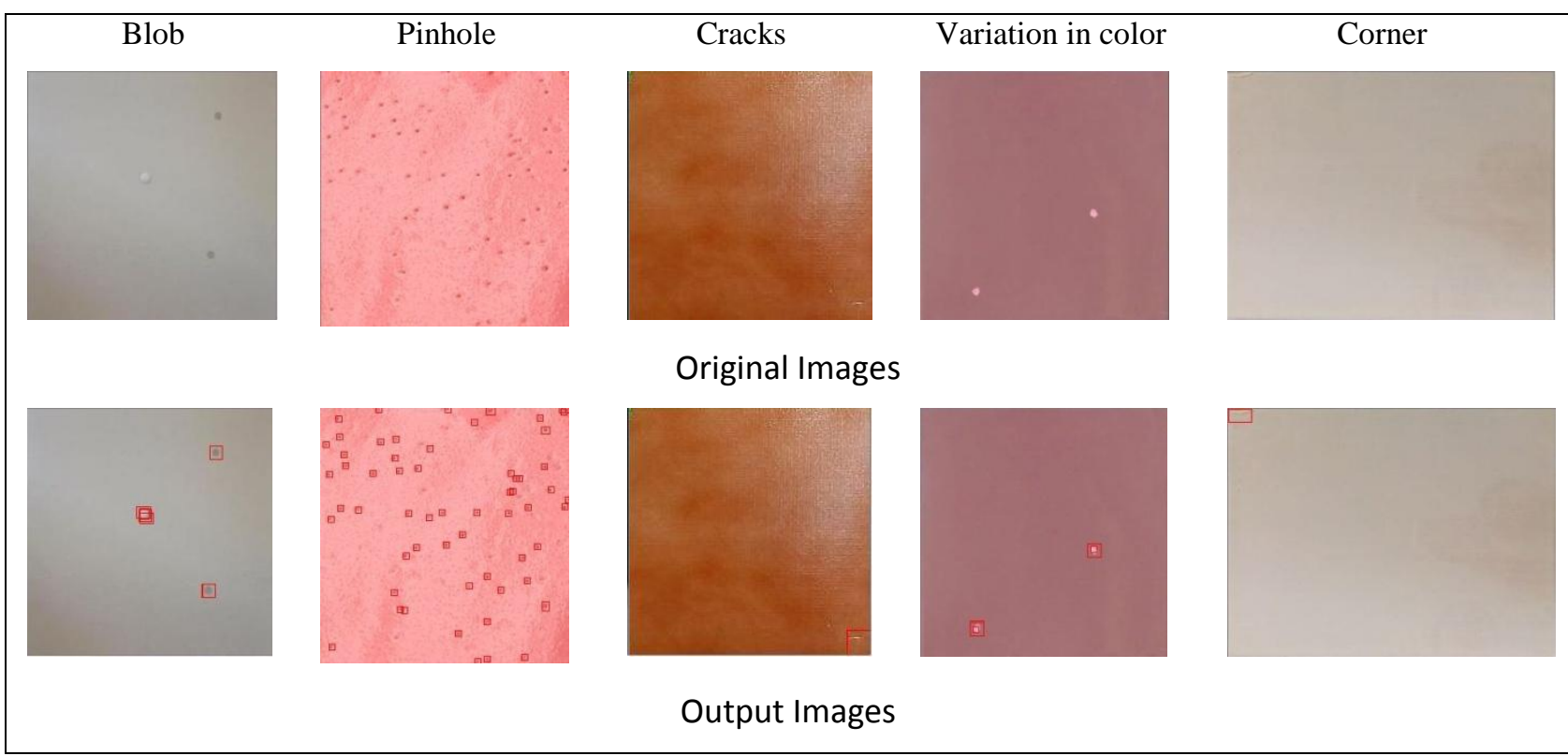

\section{CONCLUSION}

To detect object from the image, specific edge detection technique can be used by considering effect of external environment. Preprocessing step requires contrast stretching which is fulfilled by histogram equalization. Some methods for detection of object give better results but consume more time which is not acceptable in real time environment. Whereas using morphology operation, smoothing, noise reduction, detection of object can be done in less time. For detecting defect(s) in corner, more effort is required as compared to detecting other defects. Thus we can conclude that no such algorithm has proposed through which we can find all the defects at a time. In future, one generalized algorithm can be prepared to find all types of defects.

\section{REFERENCES}

[1] Garcia-Alcaraz, Jorge Luis, Maldonado-Macias, Aide Aracely, Cortes-Robles, Guillermo, Latin AmericaSpringer, 2014. "Lean Manufacturing in the Developing World_ Methodology, Case Studies and Trends".

[2] Rashmi Mishra, Dolly Shukla , International Journal of Emerging Technology and Advanced Engineering, Volume 4, March 2014. "An Automated Ceramic Tiles Defect Detection and Classification System Based on Artificial Neural Network".

[3] Sunpreet Singh, Maninder Kaur, IOSR Journal of Engineering, Volume 2(3), pp 429-32, March 2012. "Machine Vision System for Automated Visual Inspection of Tile's Surface Quality".

[4] Rafael C. Gonzalez and Richard E. Woods, third edition, Pearson Education International. "Digital Image Processing".
[5] Gonzalez, JM Valiente, F. Acebron Linuesa, and F. Lopez Garcia, 2010. "An automatic visual inspection system for ceramic tile manufacturing defects".

[6] Yadraj Meena, Dr. Ajay Mittal, International Journal of Advanced Research in Computer Science and Software Engineering, Volume 3, July 2013. "Blobs and Cracks Detection on Plain Ceramic Tile Surface".

[7] Costas Boukouvalas et al., DIBE, University of Genoa, 16145 Genova, Italy, pp. 49-54. ," An Integrated System for Quality Inspection of Tiles".

[8] Dr. S. Vijayarani, Mrs. M. Vinupriya, International Journal of Innovative Research in Computer and Communication Engineering, Volume 1, October 2013. "Performance Analysis of Canny and Sobel Edge Detection Algorithms in Image Mining".

[9] Vineet Saini, Rajnish Garg, IOSR Journal of Electronics and Communication Engineering, Volume 1, May-June 2012, ISSN: 2278-2834. "A Comparative Analysis on Edge Detection Techniques Used in Image Processing".

[10] Radhika Chandwadkar, Proceedings of Sixth IRAJ International Conference, Pune, India, October 2013. "Comparison Of Edge Detection Techniques".

[11] Elbehiery, H., A. Hefnawy, and M. Elewa. International Journal of Computer, Information Science and Engineering, , pp. 158-162, 2007. "Surface Defects Detection for Ceramic Tiles Using Image Processing and Morphological Techniques."

[12] Nenadic, K., Igor Novak, Josip Job, F. Jovic, and Z. Jagnjic, 48th International Symposium ELMAR-2006, Zadar, Croatia, pp. 103-106, 07-09 June 2006. “A Possibility of Applying Differential Digital Holography in Manufacturing Process". 